\title{
ASPECTOS ECOLÓGICOS DA LEISHMANIOSE TEGUMENTAR AMERICANA 9. Prevalência/incidência da infecção humana nos municípios de Pedro de Toledo e Miracatu, Sáo Paulo, Brasill(1)
}

\author{
Almério de Castro GOMES(2), Yoshimi Imoto YAMAMOTO(3), Antonio Norberto CAPINZAIKI(4), \\ Neusa Maria Moreira AMARAL(4) \& Alberto Jorge Garcia GUIMARÄES(3).
}

\section{RESUMO}

Este trabalho apresenta aspectos epidemiológicos da leishmaniose tegumentar nos municípios de Pedro de Toledo e Miracatu, região do Vale do Ribeira, estado de São Paulo, referente ao período 1973-1984. Foi feita uma análise retrospectiva de 108 e 65 casos humanos, respectivamente, para os dois municípios acima. Outro estudo prospectivo foi realizado através do exame clínico e testes de Montenegro, imunofluorescência indireta (IF) e hemaglutinação passiva (HA). Duzentos e setenta e três pessoas foram examinadas, sendo que 22 tinham leishmaniose clínica; 10,2 e $12,8 \%$ foram soropositivos à IF e $\mathrm{HA}$, respectivamente. $\mathrm{O}$ teste de Montenegro foi aplicado em 154 indivíduos residentes em Pedra do Largo, com prevalência de $25,5 \%$ de infeç̧ão humana. Destes, 5,8\% eram crianças com idade entre 0 e 9 anos. A morbidade mostrou-se variável no tempo, manifestada sob a forma de surtos epidêmicos explosivos e sugerindo feição cíclica ainda mal definida. Notou-se tendência para incidência nula em período subseqüente a cada surto. $O$ aspecto epidemiológico geral mostra uma leishmaniose tegumentar com baixa endemicidade. O padrão de transmissão pareceu não depender do contato do homem com a floresta e a infeç̧ão humana sem distinção entre crianças e adultos.

UNITERMOS: Leishmaniose tegumentar, epidemiologia, prevalência, incidência e testes imunológicos.

\section{INTRODUÇĀO}

No Estado de São Paulo a leishmaniose tegumentar tem sido endêmica desde o início do século. Entretanto, a região do Vale do Ribeira foi considerada área de baixa endemia ou indene por muitas décadas passadas (PESSOA \& PESTA$\left.\mathrm{NA}^{25}, 1940\right)$. FORATTINI \& OLIVEIRA ${ }^{6}$ (1957) descreveram o primeiro foco dessa parasitose no municipio de Jacupiranga, pertencente à mesma região.

No Panamá, as relações do homem com a floresta e ambiente modificado, o comportamento da incidência segundo atributos pessoais como idade, sexo e ocupação; a distribuição dos casos humanos no tempo e espaço, sugeriram padrōes epidemiológicos diferenciados da leishmaniose tegumentar americana (HERRER \& CHRISTENSEN ${ }^{16,17}$ e HERRER et al. $\left.{ }^{18}, 1976\right)$, os quais têm sido também observados em outras localidades da América Central e do Sul.

Este quadro, contudo, tende a se apresentar não uniforme, mas dependente das características ecológicas de cada região, do poder infectivo das leishmanias, dos hábitos dos flebotomíneos e de barreira imunológica representada pelo estado de imunidade dos indivíduos habitantes das áreas endêmicas.

Na região do Vale do Ribeira, a leishmaniose tegumentar tem apresentado feiçðes epidemiológicas distintas dos padrões do Panamá,

(1) Trabalho financiado pelo $\mathrm{CNPq}$ - Processos 4455/81 e 405737/82.

(2) Departamento de Epidemiologia da Faculdade de Saúde Pública, Universidade de São Paulo. Av. Dr. Amaldo, 715.01255 - São Paulo, SP. Brasil.

(3) Departamento de Análises Clínicas e Toxicológicas da Faculdade de Ciências Farmacêticas da Universidade de Sāo Paulo. Caixa Postal, 30786. Sāo Paulo - SP. Brasil.

(4) Departamento de Saúde do Vale (SUS). Município de Registro, São Paulo - SP, Brasil. 
GOMES, A. de C.; YAMAMOTO, Y.I.; CAPINZAIKI, A.N.; AMARAL, N.M.M. \& GUIMARÃES, A.J.G. - Aspectos ecológicos da leishmaniose tegumentar americana. 9. Prevalência/incidência da infeç̧ão humana nos municípios de Pedro de Toledo e Miracatu, São Paulo, Brasil. Rev. Inst. Med. trop. S. Paulo, 34(2): 149-158, 1992.

a começar pela maneira como a doença se manifestou endemicamente, ou seja, muitas décadas após o homem tê-la colonizado e, sobretudo, quando não mais existia a floresta primária dos níveis topográficos mais baixos. Esta característica, ao lado da suposta baixa infecciosidade da floresta perenifólia higrófila do Sistema da Serra do Mar na região (GOMES \& GALATI ${ }^{11}, 1989$ ), tem fundamentado a hipótese de transmissão extraflorestal da leishmaniose tegumentar. Ademais, embora esta doença grasse sobretudo em regiões de colonização recente, não existe ainda nenhuma evidência que esse padrão epidemiológico tenha ocorrido, se quer uma vez, durante os desflorestamentos levados a efeito na referida região.

Por ocasião dos estudos entomológicos desenvolvidos no Vale do Ribeira, pôde-se registrar que Lutzomyia intermedia, Lutzomyia migonei e Lutzomyia fischeri mantiveram freqüência regular no domicílio (GOMES et al. ${ }^{12}$ 1986). No ambiente florestal, Psychodopygus ayrozai foi quase a única espécie silvestre em isca humana, todavia, sua baixa antropofilia e comparecimento esporádico nas coletas, igualmente ao que ocorreu com as três espécies anteriores, não mereceu estar incluída no rol das espécies suspeitas de serem vetoras nesse tipo de ambiente.

Completando estudos descritivos seriados sobre os principais elos da cadeia epidemiológica da doença no Vale do Ribeira, o presente trabalho tem por objetivo avaliar a condição de risco para ocorrência e evidenciação do padrão epidemiológico da infecção humana. Para tanto, foi feita uma análise retrospectiva e prospectiva, a partir de notificações dos casos, exame clínico e testes imunológicos.

\section{MATERIAL E MÉTODOS}

A região estudada foi descrita por FORATTINI et al. ${ }^{8}$ (1978) e complementada em seus aspectos peculiares por GOMES et al. ${ }^{13}$ (1990). Os municípios de Miracatu e Pedro de Toledo são limítrofes e têm topografia acidentada devido aos prolongamentos das ondulações a leste pela Serra de Paranapiacaba e a oeste pela Serra dos Itatins. A zona rural está dispersa entre os vales onde a vegetação primitiva foi substituída pelo cultivo da banana e outras culturas de subsistência. Aliás, as habitações daqui estão sempre próximas das culturas ou de resíduos esparsos de vegetação secundária cujos indivíduos arbóreos de maior porte são em número restrito, enquanto que a mata preservada está mais distante (GOMES et al. ${ }^{13}$, 1990).

Como fatores antecedentes da região, que justificaram a escolha dos dois municípios em questão, estão as características típicas de zona rural, persistência da doença, surto epidêmico ocorrido anteriormente e durante o ano de 1981. Nessa ocasião e durante o primeiro ano de investigações, foram isoladas algumas cepas de L. (Viannia) braziliensis de casos humanos e caninos, sendo que uma parte delas foi relatada por MACHADO \& MILDER ${ }^{21}$ (1986). A tripanossomiase americana não é conhecida nos dois municípios e a infecção humana na região constitui fator raro e não transmitida via triatomíneo (FORATTINI et. al. ${ }^{9}, 1980$ ).

As localidades estudadas foram os bairros Pedra do Largo, do Município de Miracatu, e São Lourencinho e Vale do Kiri, de Pedro de Toledo, os quais eram contíguos e localizados à margem do Rio São Lourencinho.

Um recenseamento domiciliar foi realizado nos meses de fevereiro/março de 1982. Nesta ocasião foram registrados atributos pessoais, tais como, nome, sexo, idade, tempo de permanência no bairro e história clínica de leishmaniose. $\mathrm{Na}$ população estudada foram realizados exames clínicos e coletas de sangue para realização de testes sorológicos.

Para o estudo prospectivo baseado na incidência foi formado um grupo de indivíduos, do bairro Pedra do Largo, composto de várias idades e sexo. Este grupo foi acompanhado durante três anos ininterruptos. A escolha deste bairro se deveu ao conglomerado de habitações, maior receptividade de seus moradores, cuja maioria possuía residência própria.

Exame clínico: os indivíduos recenseados foram submetidos ao exame clínico para confirmação do registro presente e passado da infecção, através de busca das cicatrizes compatíveis com leishmaniose cutânea e ou lesões recentes. No grupo de Pedra do Largo, o exame clínico foi sistemático a cada dois meses, no período de 1982 a 1984. $O$ intervalo entre os exames foi baseado na suposição de que o período médio de incubação da doença na região poderá estar em torno de 60 dias (informação pessoal de Dr. Capinzaiki).

Inquérito sorológico: a primeira coleta de 
sangue foi realizada nos meses de fevereiro/março de 1982, a qual envolveu os indivíduos dos três bairros já referidos. Incluíram nessa amostra todos os indivíduos com e sem história de leishmaniose. As coletas dos anos de 1983 e 1984 envolveram somente o grupo formado no bairro Pedra do Largo. Para a reação de imunofluorescência indireta (IF) seguiu-se a técnica descrita por GUIMARÃES et al. ${ }^{14,15}$ (1974 e 1990), empregando como antígeno formas promastigotas de L. major-like (MHOM/BR/71/49), obtida do meio de LIT (triptose e infusão de fígado). O conjugado fluorescente utilizado foi anti-IgG humana (Travenol Laboratories-USA). As leituras foram feitas em microscópio de fluorescência NIKON, sistema de transiluminação, com lâmpada de mercúrio $\mathrm{SH}$ 200 , filtro de excitação B e de barreira $515 \mathrm{~W}$. Soro padrão positivo e negativo foram sempre incluídos no teste para controle da especificidade. O limiar de reatividade escolhido foi a diluição $1 / 40$ do soro, com base no relato de SOUZA et al. ${ }^{28}$ (1982). Reação de hemaglutinação passiva (HA) - o reagente de hemaglutinação foi preparado a partir do extrato protéico de L. major-like (MHOM/BR/ 71/49) e os soros foram processados conforme a técnica descrita anteriormente (YAMAMOTO et al. ${ }^{30}$, 1981). Para a detecção de eventuais aglutininas inespecíficas, todos os soros foram testados concomitantemente com hemácias utilizadas na preparação do reagente. Foi considerado como limiar de reatividade a diluição de 1/32.

Inquérito intradérmico (IDR): foi utilizado o antígeno de Leishmania produzido pela UFMG, contendo 40 $\mathrm{\mu g} / \mathrm{ml}$ (MELO et al. ${ }^{22}$, 1977) e cedido gentilmente pelo professor Mayrink. Este antígeno foi inoculado no antebraço, volume 0,1 $\mathrm{ml}$ e a leitura da reação feita após 72 horas. Foi considerada reação positiva a enduração com diâmetro igual ou superior a $5 \mathrm{~mm}$. Nos anos de 1983 e 1984 foram realizados inquéritos exclusivamente no grupo de Pedra do Largo. Os indivíduos positivos no primeiro teste foram excluidos do segundo. Não foi aplicado o teste de Montenegro nos indivíduos de São Lourencinho e Vale do Kiri, porque um outro antígeno inicialmente utilizado apresentou problema de antigenicidade. Sua substituição imediata não foi possivel, face ao retardamento na aquisição do antígeno produzido pela UFMG.

Incidência: os casos humanos de leishmaniose tegumentar registrados a cada ano serviram para determinar os coeficientes de incidência. Esses dados e a situação demográfica dos municípios de
Pedro de Toledo e Miracatu foram fornecidos pelo SISTEMA UNIFICADO DE SAÚDE - SUS $^{49} \mathrm{Mu}$ nicípio de Registro. O cálculo dos coeficientes foi baseado em LAURENTI et al. ${ }^{19}$ (1985).

Prevalência: foi determinada a partir dos resultados da IDR e acrescida de informaçðes dos testes sorológicos.

\section{RESULTADOS}

O recenseamento realizado em 1982 abrangeu 73 habitações humanas e contingente de 355 pessoas, sendo que os participantes do estudo foram apenas $273(76,9 \%)$ (Tabela 1). Os outros 82 restantes deixaram de tomar parte nesta investigação por estarem ausentes nos dias trabalhados, bem como os recém-nascidos e os que recusaram o exame clínico ou a doação de sangue.

O número de pessoas que formaram o grupo de estudo prospectivo em Pedra do Largo foi de

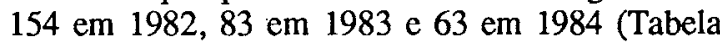
3 ), ficando assim configurada uma perda por evasão de indivíduos da área.

O exame clínico específico mostrou que do total de 273 pessoas, 22 apresentavam história e ou cicatrizes compatíveis com leishmaniose (Tabela 1). Uma parte desses pacientes já havia tido cura antimonial. Onze pacientes haviam contraído a doença durante o surto de Miracatu em 1981 e ainda encontravam-se em fase final de tratamento quando foram coletadas amostras de sangue; quatro haviam tido leishmaniose nos anos de 1973, 1976, 1979 e 1980, sendo que todos residiram sempre nos bairros de São Lourencinho e Kiri, em Pedro de Toledo; quatro não souberam informar quando haviam tido a doença, mas sempre residiram na área e três outros restantes eram procedentes de localidades fora do Vale, estes não souberam informar há quanto tempo haviam tido a doença. Finalmente, 251 indivíduos não apresentaram nenhum sinal ou história de leishmaniose cutânea.

Os testes sorológicos nos bairros de Pedra do Largo, São Lourencinho e Vale do Kiri foram realizados em 273 pessoas, com idades entre 1 e 87 anos, sendo que 134 pertenciam ao sexo masculino e 139 ao feminino. O grupo etário de 0 a 9 anos representou $30,7 \%$ do global examinado. Os resultados positivos das reaçð̄es de IF $(10,2 \%)$ e HA $(12,8 \%)$ constam da Tabela 2 . Nela se observa a positividade ocorrer em todas as faixas etárias, in- 
GOMES, A. de C.; YAMAMOTO, Y.I.; CAPINZAIKI, A.N.; AMARAL, N.M.M. \& GUTMARÃES, A.J.G. - Aspectos ecológicos da leishmaniose tegumentar americana. 9. Prevalência/incidência da infeç̧ão humana nos municípios de Pedro de Toledo e Miracatu, São Paulo, Brasil. Rev. Inst. Med. trop. S. Paulo, 34(2): 149-158, 1992.

Tabela 1

Número de pessoas recenseadas, número de pessoas examinadas e prevalência de portadores de lesões ativas ou cicatrizes, segundo o sexo, idade e localidades de Pedro de Toledo e Miracatu, 1982.

\begin{tabular}{lcccccccc}
\hline Localidades & Idade & \multicolumn{3}{c}{ Recenseada } & \multicolumn{3}{c}{ Examinada } & Com lesões ativas ou \\
& & Masc. & Fem. & Total & Masc. Fem. & Total & cicatrizes \\
\hline Pedra do Largo & $0-9$ & 27 & 32 & 59 & 23 & 26 & 49 & - \\
& $10-34$ & 37 & 36 & 73 & 34 & 35 & 69 & 7 \\
& $35 \mathrm{e}+$ & 22 & 18 & 40 & 20 & 16 & 36 & 3 \\
São Lourencinho & $0-9$ & 19 & 11 & 30 & 9 & 8 & 17 & - \\
& $10-34$ & 18 & 18 & 36 & 12 & 14 & 26 & 5 \\
& $35 \mathrm{e}+$ & 18 & 15 & 33 & 13 & 10 & 23 & 1 \\
Vale do Kiri & $0-9$ & 22 & 12 & 34 & 11 & 7 & 18 & 1 \\
& $10-34$ & 23 & 16 & 39 & 9 & 16 & 25 & 4 \\
& $35 \mathrm{e}+$ & 4 & 7 & 11 & 3 & 7 & 10 & 1 \\
\hline TOTAL DE & $0-9$ & 68 & 55 & 123 & 43 & 41 & 84 & 1 \\
LOCALIDADES & $10-34$ & 78 & 70 & 148 & 55 & 65 & 120 & 16 \\
& $35 \mathrm{e}+$ & 44 & 40 & 84 & 36 & 33 & 69 & 5 \\
\hline TOTAL & Geral & 190 & 165 & 355 & 134 & 139 & 273 & 22 \\
\hline
\end{tabular}

Tabela 2

Número e Percentagem de indivíduos segundo positividade do inquérito sorológico baseado na imunofluorescência indireta (IF) e hemaglutinação passiva (HA), de acordo com a idade e o sexo, município de Pedro de Toledo e Miracatu, 1982.

\begin{tabular}{lcrrrr}
\hline & & \multicolumn{3}{c}{ POSITIVIDADE } \\
Faixa etária & Sexo & \multicolumn{2}{c}{ IF } & \multicolumn{2}{c}{ HA } \\
\hline $0-9$ & $\mathrm{M}$ & $4,6 \%$ & $(2 / 43)$ & $9,3 \%$ & $(4 / 43)$ \\
& $\mathrm{F}$ & $9,7 \%$ & $(4 / 41)$ & $17,1 \%$ & $(7 / 41)$ \\
$10-34$ & $\mathrm{M}$ & $7,2 \%$ & $(4 / 55)$ & $9,1 \%$ & $(5 / 55)$ \\
& $\mathrm{F}$ & $16,9 \%$ & $(11 / 65)$ & $21,5 \%$ & $(14 / 65)$ \\
$35 \mathrm{e}+$ & $\mathrm{M}$ & $8,3 \%$ & $(3 / 36)$ & $8,3 \%$ & $(3 / 36)$ \\
& $\mathrm{F}$ & $12,1 \%$ & $(4 / 33)$ & $6,1 \%$ & $(2 / 33)$ \\
\hline Total & & $10,2 \%(28 / 273)$ & \multicolumn{2}{c}{$12,8 \%(35 / 273)$} \\
\hline
\end{tabular}

clusive no grupo de 0 a 9 anos, a qual foi comparável com a de outros grupos. Da mesma forma, não se observou diferença significativa $(=0,05)$ entre homens e mulheres).

Do grupo de 251 indivíduos sem leishmaniose, 51 apresentaram sorologia positiva pela IF, HA ou ambas. Verificando a ficha de seus registros constatou-se que 31 deles residiram sempre na área estudada e outros 20 tiveram procedências diversas, porém sem indicação de serem oriundos de área chagásica. Dezessete destes tinham tempo de residência inferior a dois anos.

Os inquéritos sorológicos levados a efeito em Pedra do Largo, nos anos de 1982, 1983 e 1984, têm seus resultados na Tabela 3, onde se constatou uma redução na positividade sorológica, por evasão de indivíduos ou negativação de título. A variação percentual de indivíduos não reagentes a IF, na amostra estudada em 1982 , foi de $84,4 \%$ e de $95,2 \%$ em 1984, numa amostra populacional respectiva de 154 e 63 pessoas.

Quanto ao teste intradérmico de Montenegro, $25,5 \%$ das pessoas examinadas encontravam-se positivas em Pedra do Largo (Tabela 4), sendo que $8(9,3 \%)$ foram doentes diagnosticados e tratados e $14(16,3 \%)$ não tinham sintomas clínicos ou história de leishmaniose. Do grupo não reator, os 30 que foram submetidos a um segundo exame em 1984, continuaram sendo negativos. Quanto ao 
GOMES, A. de C.; YAMAMOTO, Y.I.; CAPINZAIKI, A.N.; AMARAL, N.M.M. \& GUIMARÃES, A.J.G. - Aspectos ecológicos da leishmaniose tegumentar americana. 9. Prevalência/incidência da infecção humana nos municípios de Pedro de Toledo e Miracatu, São Paulo, Brasil. Rev. Inst. Med. trop. S. Paulo, 34(2): 149-158, 1992.

Tabela 3

Distribuição percentual dos indivíduos positivos através da Imunofluorescência indireta (IF) e Hemaglutinaçăo Passiva (HA), segundo a idade e ano, Pedra do Largo, Miracatu, período de 1982/84.

\begin{tabular}{|c|c|c|c|c|c|c|}
\hline \multirow{2}{*}{$\begin{array}{l}\text { Grupo } \\
\text { Etário }\end{array}$} & \multicolumn{2}{|c|}{1982} & \multicolumn{2}{|c|}{1983} & \multicolumn{2}{|c|}{1984} \\
\hline & IF & HA & IF & $\mathrm{HA}$ & IF & HA \\
\hline $0-9$ & $12,2 \%(6 / 49)$ & $16,3 \%(8 /$ & $15,0 \%(3 / 20)$ & $(0 /$ & $-\quad(0 / 15)$ & $6,7 \%$ \\
\hline $10-34$ & $18,8 \%($ & $13,0 \%$ & $7,1 \%(3 / 42)$ & $4,8 \%(2 / 42)$ & $6,7 \%(2 / 30)$ & $3,3 \%(1 / 30)$ \\
\hline $35 \mathrm{e}+$ & $13,9 \%(5 / 36)$ & $2,8 \%(1 / 36)$ & - $\quad(0 / 21)$ & $(0 / 21)$ & $5,5 \%(1 / 18)$ & $\begin{array}{l}-\quad(0 / 18) \\
\end{array}$ \\
\hline Total & $15,6 \%(24 / 154)$ & $11,7 \%(18 / 154)$ & $7,2 \%(6 / 83)$ & $2,4 \%(2 / 83)$ & $4,8 \%(3 / 63)$ & $3,2 \%(2 / 63)$ \\
\hline
\end{tabular}

Tabela 4

Distribuição percentual dos indivíduos positivos através da intradermorreação de Montenegro (IDR), segundo a idade, sexo e ano. Pedra do Largo, Miracatu 1983/84.

\begin{tabular}{|c|c|c|c|c|}
\hline F.etária & masculino & $\begin{array}{l}1983 \\
\text { feminino }\end{array}$ & total & 1984 \\
\hline $\begin{array}{c}0-9 \\
10-34 \\
35 \mathrm{e}+\end{array}$ & $\begin{array}{ll}27,3 \% & (3 / 11) \\
28,6 \% & (6 / 21) \\
41,7 \% & (5 / 12)\end{array}$ & $\begin{array}{l}18,2 \%(2 / 11) \\
15,0 \%(3 / 20) \\
27,3 \%(3 / 11)\end{array}$ & $\begin{array}{ll}22,8 \% & (5 / 22) \\
21,9 \% & (9 / 41) \\
34,8 \% & (8 / 23)\end{array}$ & $\begin{array}{l}-(0 / 9) \\
-(0 / 13) \\
-\quad(0 / 8)\end{array}$ \\
\hline Total & $31,8 \%(14 / 44)$ & $19,0 \%(8 / 42)$ & $25,5 \%(22 / 86)$ & $-(0 / 30)$ \\
\hline
\end{tabular}

atributo sexo, verificou-se que o masculino predominou sobre o feminino, mas sem apresentar diferença significante. Quanto à idade, se assinalou que a distribuição dos resultados da sorologia e teste intradérmico sinalizaram para uma positividade equivalente entre os grupos etários estudados.

O título de anticorpos detectados pela IF e HA foi relativamente baixo, sendo a média geométrica (MGT) de 42 a 43, respectivamente. Quanto à reação de Montenegro, houve variação entre 5 e $20 \mathrm{~mm}$ de enduração, sendo que a média foi de $8,9 \mathrm{~mm}$.

Na Tabela 5 estão expostos os coeficientes de incidência da leishmaniose, distribuídos segundo o sexo e idade, no triênio 1979/81. Pedro de Toledo, como área de segunda ocorrência do surto epidêmico, evidenciou destacadamente a doença entre os indivíduos do sexo feminino. Miracatu, como área de primeira ocorrência de um surto, mostrou coeficientes com valores muito próximos entre si. No ano de 1981 ocorreram coeficientes de 68,6 e 59,7 por 10.000 habitantes, em crianças com idade de 0 a 9 anos. Tal registro pareceu bem expressivo.

$\mathrm{Na}$ Tabela 6 estão registrados os coeficientes de incidência da leishmaniose tegumentar no município de Miracatu. Embora o registro da doença aqui tenha ocorrido a partir de 1979 , somente em 1981 foi registrado o pico máximo da morbidade, ou seja, 19,7 casos por 10.000 habitantes. A distribuição etária de todos os casos notificados nesse município evidencia valores muito próximos entre si enquanto a distribuição temporal mostra concentração dos casos no triênio 1979/81.

Na Tabela 7 estão os coeficientes de incidência ocorridos entre 1973 e 1983 . O mais elevado pico de morbidade ocorreu no primeiro surto epidêmico e foi de 71,5 casos por 10.000 habitantes. Além disso, os coeficientes mais significativos se concentraram nos triênios 1973/75 e 1979/81. Portanto, verificou-se que cada surto epidêmico foi sucedido pelo declínio da incidência, a qual chegou a ser até nula em 1977, 1978, 1982 e 1984. Quanto à distribuição etária dos casos, notou-se acometimento da doença em indivíduos de todas as idades, porém se ressalta coeficientes bem significativos, i.é., de 136,121 e 80,1 por 10.000 habitantes, nas faixas respectivas de 10 a 14,15 a 29 e 5 a 9 anos. Os demais resultados mostraram-se muito próximos entre si, exceto o de 43,7 por 10.000 habitantes verificado na faixa 60 e mais. No triênio 1979/81 a distribuição dos coeficientes segundo o sexo foi predominante para o feminino em Pedro de Toledo, enquanto os valores em Miracatu foram equivalentes para os dois sexos. 


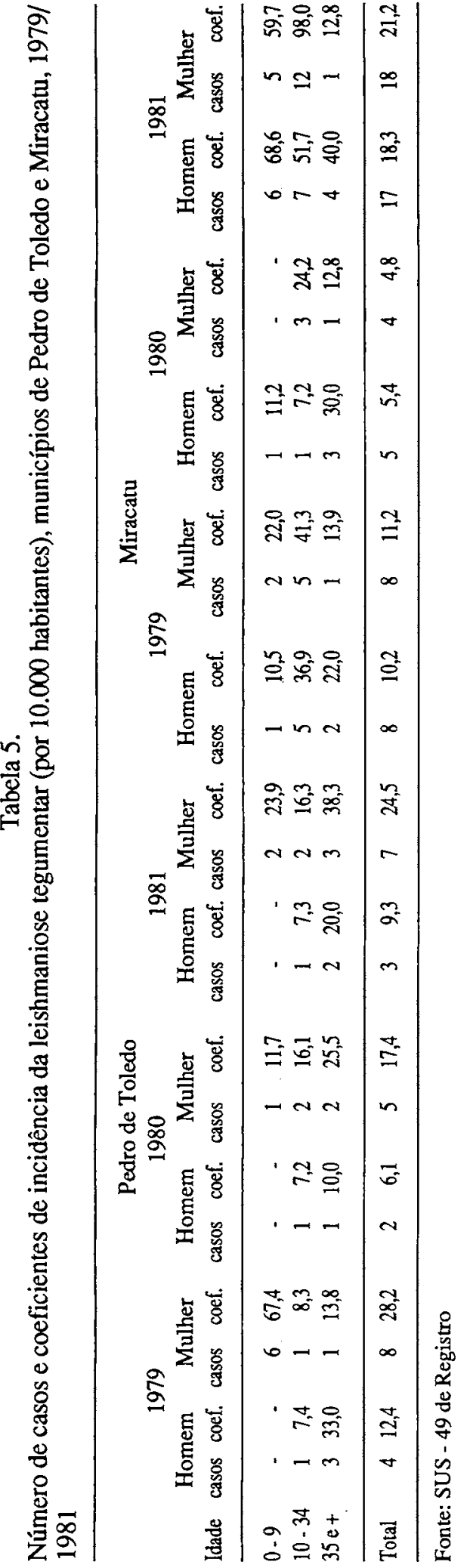

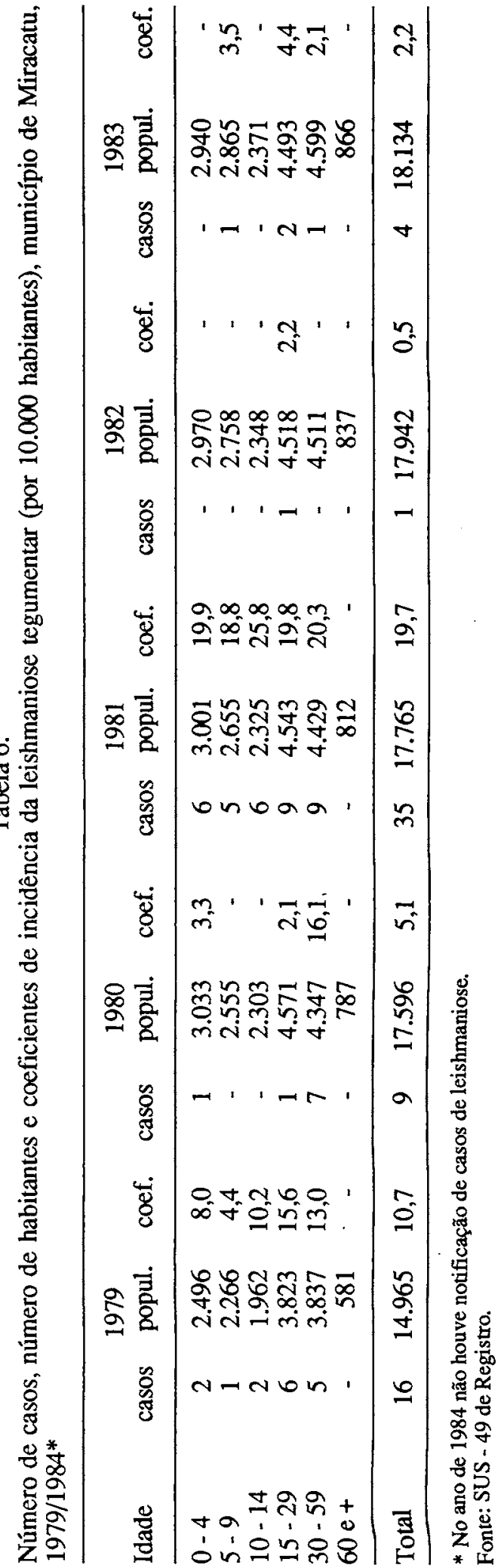




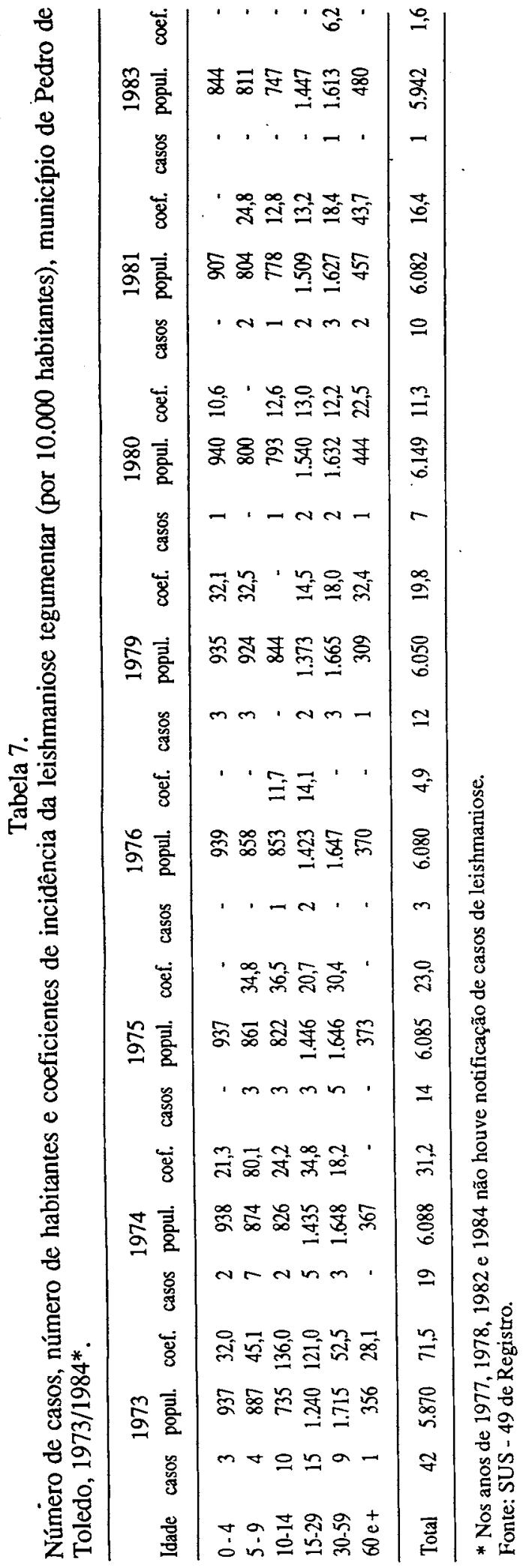

\section{DISCUSSĀO}

O Estado de São Paulo foi pioneiro na definição do padrão epidemiológico da leishmaniose tegumentar americana durante os surtos epidêmicos associados à derrubada das florestas virgens (FORATTINI', 1973).

Os municípios de Pedro de Toledo e Miracatu, onde registros epidêmicos vêm ocorrendo desde 1973 (FORATTINI et al.?, 1973, e ROCHA E SILVA et al. $\left.{ }^{26}, 1980\right)$ sediaram o presente estudo. Contrariando aquele aspecto epidemiológico esperável, não há nos dois municípios qualquer registro ou história de ocorrência de desflorestamento simultâneo ou próximo ao aparecimento da doença. Além do mais, os censos demográficos do período 1970-1980 indicaram que o Vale do Ribeira comportou-se como área de evasão populacional (RODRIGUES ${ }^{27}, 1982$ ). Durante este estudo, verificou-se migrações freqüentes entre as comunidades rurais da região, haja visto a perda de 91 indivíduos do grupo de estudo prospectivo.

Do ponto de vista epidemiológico, a endemia regional vem sendo mantida graças à ocorrência de casos isolados ou agrupados, distribuídos esparsamente no tempo e espaço, como se a transmissão se processasse sob condições transitórias.

Analisando a morbidade por leishmaniose em Miracatu e Pedro de Toledo, verificou-se que os surtos epidêmicos tiveram duração trianuais (Tabelas 6 e 7). Entretanto, após cada um ocorreu redução sensível da incidência, tornando até nula. Tal aspecto sugeriu incidência com caráter explosivo e com baixo comprometimento da população exposta. O nivel da morbidade, no entanto, mostrou-se variável entre os eventos, posto que em Pedro de Toledo a maior taxa ocorreu no primeiro ano do aparecimento da doença, ou seja, 71,5 por 10.000 habitantes em 1973, enquanto em Miracatu foi de 19,7 por 10.000 habitantes, porém correspondendo ao final do surto epidêmico, i.é., 1981. Este aspecto mostra existência de fatores locais influenciando a morbidade da doença. Por outro lado, se for levado em conta a ausência de casos clínicos e de infecções humanas, sucedente ao surto do triênio 1979/81 (Tabela 4), ratifica-se ausência de transmissão ativa do parasita, não sabendo-se como isto ocorre. Aliás, este quadro evidencia uma certa feição cíclica da doença, a qual somente futuros estudos poderão melhor elucidá-la. Retornando a análi- 
GOMES, A. de C.; YAMAMOTO, Y.I.; CAPINZAIKI, A.N.; AMARAL, N.M.M. \& GUIMARÃES, A.J.G. - Aspectos ecológicos da leishmaniose tegumentar americana. 9. Prevalência/incidência da infeç̧ão humana nos municípios de Pedro de Toledo e Miracatu, São Paulo, Brasil. Rev. Inst. Med. trop. S. Paulo, 34(2): 149-158, 1992.

se da morbidade, o encontro de incidência anual constante de 2,3 por 1.000 habitantes na Guiana Francesa, onde a transmissão é selvática (DEDET ${ }^{4}$, 1990), e as oscilaçð̃es entre $0 / 71,5$ ou $0 / 19,7$ por 10.000 habitantes em Pedro de Toledo e Miracatu, respectivamente, fazem sugerir condiçōes estáveis e instáveis de transmissão, nas respectivas localidades.

No que concerne à idade, a distribuição dos coeficientes de incidência em Miracatu e Pedro de Toledo mostraram resultados similares, pois a grande maioria deles estiveram muito próximos entre si. Isoladamente, notou-se que os três coeficientes mais elevados ocorreram em épocas e idades diferentes, i.é., grupos etários de 5 a 9,10 a 14 e 15 a 29 , respectivamente (Tabela 7). Coeficientes mais elevados no sexo feminino que no masculino e a diferença de comportamento da incidência, quando o surto é de ocorrência primária ou não, caracterizaram bem a feição epidemiológica local (Tabela 5). Por conseguinte, tornou-se difícil destacar grupo de risco definido, o que, a priori, significa igualdade de exposição de todos os indivíduos à doença. Corrobora com este fato, os resultados semelhantes obtidos por ROCHA E SILVA et al. ${ }^{26}$ (1980), quando da análise das notificações do período epidêmico 1978/79 ocorrido no Vale do Ribeira. Em Camorim, município do Rio de Janeiro, LIMA et al. ${ }^{20}$ (1988) relataram presença da leishmaniose tegumentar nas pessoas ali residentes, independentemente da idade, sexo, ocupação e tipo de habitação, sendo que a incidência foi maior entre crianças. Não obstante a similaridade na distribuição dos casos humanos entre as duas áreas, deve-se lembrar que a doença em Camorim está relacionada a foco residual de enzootia silvestre, conforme define FORATTINI ${ }^{5}$ (1973), enquanto que, em Pcdro de Toledo e Miracatu, o foco enzoótico está relacionado ainda ao ambiente florestal primário.

A proporção de 10,2 e $12,8 \%$ de positividade a IF e HA, respectivamente, pouco contribuiu para o conhecimento da prevalência da doença nas três comunidades estudadas. Certamente, as taxas encontradas estão influenciadas pela conduta terapêutica antimonial (MENDONÇA et al. ${ }^{23}, 1988$ ) e WALTON et al. $\left.{ }^{31}, 1972\right)$. Mesmo assim, a distribuição dos resultados, segundo a idade e o sexo, não deixa de concordar com a tendência declinante da morbidade ou ausência de novos casos. Além disso, chamou atenção o fato da média geométrica do título da IF (MGT $=42$ ) ter sido próxima à obtida por CUBA et al. ${ }^{3}, 1984$ ), i.é., MGT $=50$, quando no primeiro caso haviam apenas 11 pacientes com leishmaniose clínica e no segundo os pacientes eram portadores de formas cutâneas, com lesð̃es simples.

Com relação ao resultado da IDR, constante da Tabela 4, observa-se que a taxa de positividade obtida em Pedra do Largo está em consonância com valores expressos por FURTADO ${ }^{10}$ (1980) bem como se assemelha ao encontrado por PESSOA \& BARRETO ${ }^{24}$ (1948), em área de desmatamento e ainda em período pequeno. Por outro lado, apesar de existirem diferença geográfica, etiológica e vetorial entre Pedra do Largo e focos de leishmaniose em comunidades do Estado de Campeche, México, a taxa de $25,5 \%$ de positividade da IDR na primeira localidade $e$ as variações entre 20 a $90 \%$ na segunda (ANDRADE-NARVAEZ et al. ${ }^{1}, 1990$ ), podem sugerir condições diferenciadas de exposição à leishmaniose cutânea. Um importante fator de diferenciação envolvido nesta analogia, poderia ser a condição de risco, baseada na diferença de infecciosidade dos dois ambientes. Mais nitida se torna a feição epidemiológica diferenciada da doença na área estudada, quando se evidencia incidência nula, por longos períodos de tempo, sem nexo cíclico com as estações climáticas mais favoráveis à transmissão, e, particularmente, quando se sabe que os indivíduos residentes em Pedro de Toledo e Miracatu mantiveram contato contínuo com as matas próximas às suas casas. Além do mais, se a causa da ausência de novos casos de leishmaniose fosse atribuída ao desaparecimento, quase por completo, dos flebotomíneos das habitações humanas e dos animais, após aplicação de DDT, como medida de controle (GOMES et al. ${ }^{13}$, 1990), mais uma vez, se excluiria o papel preponderante do ambiente florestal local, como foco de infecções humanas. Aliás, o encontro de $22,8 \%$ da IDR situarem na faixa de 0 a 9 anos e o comportamento da incidência nos dois municípios (Tabelas 4, 6 e 7), sugerem o ambiente doméstico como local mais provável de ocorrência da transmissão. Corrobora com esta hipótese a dispersão atípica de roedores denominada fenômeno da "ratada", a qual envolveu os domicílios e sobrepôs a epidemia de 1978/79 (ROCHA E SILVA et al. ${ }^{24}, 1980$ ). VILLALOBOS et al. ${ }^{29}$ (1989), assinalando $24,8 \%$ de IDR em menores de 5 anos em Zipayare, Estado de Zulia, Venezuela, suspeitaram mudança no comportamento da espécie vetora, de modo a justificar a transmissão domiciliar da leishmaniose cutânea nessa localidade. A questão da capacidade vetorial expressa por GOMES et al. ${ }^{12}$ (1986) e GOMES \& GALATI ${ }^{11}$ (1989), também concorrem 
GOMES, A. de C.; YAMAMOTO, Y.I.; CAPINZAIKI, A.N.; AMARAL, N.M.M. \& GUIMARAES, A.J.G. - Aspectos ecolбgicos da leishmaniose tegumentar americana. 9. Prevalência/incidência da infecçāo humana nos municípios de Pedro de Toledo e Miracatu, São Paulo, Brasil. Rev. Inst. Med. trop. S. Paulo, 34(2): 149-158, 1992.

para alicerçar o mesmo ponto de vista. Da mesma forma, o não encontro de IDR positiva entre menores de 5 anos em Três Braços, Bahia (BARRETO et al. $\left.{ }^{2}, 1981\right)$, significa manutenção de transmissão com caráter ainda selvático.

Face aos diferentes fatores evocados para esta análise epidemiológica, parece claro que a transmissão na área estudada é instável e direcionada ao ambiente doméstico.

\section{CONCLUSĀO}

O padrão epidemiológico da leishmaniose tegumentar na região do Vale do Ribeira indica tendência para casos isolados, que se agrupam em determinados periodos, configurando caráter explosivo da incidência. Tal feição ocorre sem evidenciar claramente a necessidade do contato do homem com a floresta. Atributos como idade, sexo e exposição à doença, sugerem local de risco dissociado do habitat natural esperado do parasito. Daí, não ter sido possível destacar grupo etário de maior risco.

\section{SUMMARY}

Ecological aspects of American Cutaneous Leishmaniasis. 9. Prevalence/incidence of the human infection in Pedro de Toledo and Miracatu municipalities, São Paulo, Brazil.

The epidemiologic study was conducted during the 1973-1984 period. The clinical prospective exam and Montenegro skin, immunoflurescent and passive hemagglutination tests have been carried out in three small localities between Pedro de Toledo e Miracatu municipalities, São Paulo, Brazil. The retrospective study of human-cases involved 108 and 65 cases registered in Pedro de Toledo e Miracatu, respectively. In the three communities studied, 273 people were examinated clinically and serologically. Twenty two individuals had had signals of cutaneous leishmaniasis; 10.2 and $12.8 \%$ were seropositive to IF and HA. Leishmanin skin testing of a sample of 154 people residents in Pedra do Largo showed prevalence of Leishmania infection in $25.5 \%$. This result involved individual of all ages and sex. However, $5.8 \%$ of them were from 0 to 9 years old. The data confirmed that active parasite transmission didn't occur every year, either. The human infection seems not to depend on man contact with a forest. The incidence relatively low suggests low endemic area for cutaneous leishmaniasis and an explosive behavior of the ca- ses. The temporal distribution of disease was irregular and the epidemiological pattern seen was different from the other endemic area of South America.

\section{AGRADECIMENTOS}

Dr. Wilson Mayrink do Instituto de Ciências Biológicas - UFMG; Dr. R. Lainson e Dr. J.J. Shaw do Wellcome Parasitology Unit-Instituto Evandro Chagas; Dra. Sabina Léa Davidson Gotlieb e Dra. Stella M. Costa Nardy da Faculdade de Saúde Pública/USP e Ida Shiama do SUS-49Registro.

\section{REFERÊNCIAS BIBLIOGRÁFICAS}

01. ANDRADE-NARVAEZ, J.; SIMMONDS-DIAZ, E.; RICO-AGUILAR, S.; ANDRADE-NARVAEZ, M.; PALOMO-CETINA, N.; CANTO-LARA, S.B.; GARCIA-MISS, M.R.; MADERA-SEVILLA, M. \& ALBERTOS-ALPUCHE, N. - Incidence of localized cutaneous leishmaniasis (chiclero's ulcer) in Mexico. Trans. roy. Soc. trop. Med. Hyg., 84: 219-220, 1990.

02. BARRETO, A.C.; CUBA, C.C.; MARSDEN, P.D.; VEXANAT, J.A. \& BELDER, M. de - Características epidemiológicas da leishmaniose tegumentar americana, em uma região endêmica do Estado da Bahia, Brasil. ILeishmaniose humana. Bol. Ofic. sanit. panamer., 90: 415-424, 1981.

03. CUBA, C.C.; BARRETO, A.C.; LLANOS-CUENTAS, E.A.; MAGALHÃES, A.V.; LAGO, E.L.; REED, S. \& MARSDEN, P.D. - Human mucocutaneous leishmaniasis in Três Braços, Bahia-Brazil: an area of Leishmania braziliensis braziliensis, transmission. 1. Laboratory diagnosis. Rev. Soc. bras. Med. trop., 17: 161-167, 1984

04. DEDET, J.P. - Cutaneous leishmaniasis in French Guiana: a review. Amer. J. trop. Med. Hyg., 43: 25-28, 1990.

05. FORATTINI, O.P. - Entomologia médica. São Paulo, Edgard Blücher, 1973. v. 4.

06. FORATTINI, O.P. \& OLIVEIRA, O. - Um foco de leishmaniose tegumentar na zona sul de São Paulo, Brasil. Arq. Fac. Hig. S. Paulo, 11: 23-34, 1957.

07. FORATTINI, O.P.; PATTOLI, D.B.G.; SERRA, O.P.; ROCHA E SILVA, E.O. \& RABELLO, E.X. - Nota sobre leishmaniose tegumentar no litoral sul do Estado de São Paulo, Brasil. Rev. Saúde públ. (S. Paulo), 7: 447-452, 1973.

08. FORATTINI, O.P.; FERREIRA, O.A.; ROCHA E SILVA, E.O. \& RABELLO, E.X. - Aspectos ecológicos da Tripanossomíase americana. XII. Variação regional da tendência de Panstrogylus megistus à domiciliação. Rev. Saúde públ. (S. Paulo), 12: 209-233, 1978.

09. FORATTINT; O.P.; ROCHA E SILVA, E.O.; BARATA, 
GOMES, A. de C.; YAMAMOTO, Y.I.; CAPINZAIKI, A.N.; AMARAL, N.M.M. \& GUIMARÃES, A.J.G. - Aspectos ecológicos da leishmaniose tegumentar americana. 9. Prevalência/incidência da infeç̧ão humana nos municípios de Pedro de Toledo e Miracatu, São Paulo, Brasil. Rev. Inst. Med. trop. S. Paulo, 34(2): 149-158, 1992.

J.M.S. \& BOAINAIN, E. - Nota sobre caso autóctone de tripanossomíase americana do litoral sul do estado de São Paulo, Brasil. Rev. Sá́de públ. (S. Paulo), 14: 143-149, 1980.

10. FURTADO, T. - Critérios para o diagnóstico da leishmaniose tegumentar americana. An. bras. Derm., 55: $81-86,1980$.

11. GOMES, A. de C. \& GALATI, E.A.B. - Aspectos ecológicos da leishmaniose tegumentar americana. 7 . Capacidade vetorial flebotomínea em ambiente florestal primário no sistema da Serra do Mar, regiāo do Vale do Ribeira, Estado de São Paulo, Brasil. Rev. Saúde públ. (S. Paulo), 23: 89-97, 1989.

12. GOMES, A. de C.; SANTOS, J.L.F. \& GALATT, E.A.B. Ecological aspects of American cutaneous leishmaniasis. 4. Observations on the endophilic behavior of the sandfly and the vectorial role of Psychodopygus intermedius in the Ribeira Valley region of the S. Paulo State Brazil. Rev. Saúde públ. (S. Paulo), 20: 280-287, 1986.

13. GOMES, A. de C.; COUTINHO, S.G.; PAIM, G.V.; OLIVEIRA, S.M.O. de; GALATI, E.A.B.; NUNES, M.P.; CAPINZAIKI, A.N.; YAMAMOTO, Y.L. \& ROTTER, P. - Aspectos ecológicos da leishmaniose tegumentar americana. 8. Avaliação da atividade enzoótica de Leishmania (Viannia) braziliensis, em ambiente florestal e peridomiciliar, regiāo do Vale do Ribeira, Estado de São Paulo, Brasil. Rev. Inst. Med. trop. S Paulo, 32: 105$115,1990$.

14. GUIMARĀES, M.C.S.; GIOVANNINI, V.C. \& CAMARGO, M.E. - Antigenic standardization for mucocutaneous leishmaniasis immunofluorescence test. Rev. Inst. Med. trop. S. Paulo, 16: 145-148, 1974.

15. GUIMARÃES, M.C.S.; CELESTE, B.J. \& FRANCO, E.L - Diagnostic performance indices for immunofluorescent test and enzyme immunoassays of leishmaniasis sera from northem and north-eastem Brazil. Bull. Wld. Hith. Org., 68: 39.43, 1990.

16. HERRER, A. \& CHRISTENSEN, A.A. - Epidemiological patterns of cutaneous leishmaniasis in Panama. I. Epidemic among small groups of settlers. Ann. trop. med. Parasit., 70: 59-65, 1976

17. HERRER, A. \& CHRISTENSEN, A.A. - Epidemiological pattems of cutaneous leishmaniasis in Panama. III. Endemic persistence of the disease. Amer. J. trop. Med. Hyg., 25: 54-58, 1976.

18. HERRER, A.; CHRISTENSEN, A.A. \& BEUMER, R.J. Epidemiological patterns of cutaneous leishmaniasis in Panama. II. Incidental occurrence of cases in non-endemic setulements. Ann. trop. Med Parasit., 70: 67-71, 1976.

19. LAURENTI, R.; MELLO JORGE, M.H.P. de; LEBRÃO, M.L. \& GOTLIEB, S.L.D. - Estatística de Saúde. São Paulo, Pedagógica e Universitária, 1985.

20. LIMA, L.C.; MARZOCHI, M.C.A.; SABROZA, P.C. \&
SOUZA, M.A. - Observações sobre a leishmaniose tegumentar, cinco anos após profilaxia. Rev. Saúde públ. (S. Paulo), 22: 73-77, 1988

21. MACHADO, M.I. \& MILDER, R.V. - Isolamento e identificação de Leishmania braziliensis de casos humanos e cães domésticos do Estado de São Paulo. R. Cent. Bioméd. Univ. Fed. Uberlândia, 2: 9-21, 1986 .

22. MELO, M.N.; MAYRINK, W.; COSTA, C.A. MAGALHÃES, P.A.; DIAS, M.; WILLIAMS, P.; ARAUJO, F.G.; COELHO, M.V. \& BATISTA, S. M. Standartization of the Montenegro antigen. Rev. Inst. Med. trop S. Paulo, 19; 161-164, 1977.

23. MENDONCA, S.C.; SOUZA, W.J.S.; NUNES, M.P. MARZOCHI, M.C.A. \& COUTINHO, S.G. - Indirect immunofluorescence test in new world leishmaniasis: serological and clinical relationship. Mem. Inst. Oswaldo Cruz, 83: 347-355, 1988.

24. PESSOA, S.B. \& BARRETTO, M.P. - Leishmaniose tegumentar americana. Rio de Janeiro, Ministério da Educação, 1948.

25. PESSOA, S.B. \& PESTANA, B.R. - Sobre a disseminação da leishmaniose tegumentar no Estado de São Paulo: resultado de um inquérito realizado nos Centros de Saúde do interior. Folha méd., 20: 20-30, 1940.

26. ROCHA E SILVA, E.O.; CAPINZAIKI, A.N.; KURATOMI, C.A. \& GUEDES, A.C. - A leishmaniose tegumentar americana no litoral do sul do Estado de São Paulo. Rev. bras. Malar., 32: 9-25, 1980.

27. RODRIGUES, R. do N. - Análise demográfica regional: região do litoral, São Paulo. São Paulo, Fundação SEADE, 1982.

28. SOUZA, W.J.S.; COUTINHO, S.G.; MARZOCHI, M.C.A.; TOLEDO, L. M. \& GOTTLIED, M.V. - Utilizaçāo da reação de imunofluorescência indireta no acompanhamento da terapêutica da leishmaniose tegumentar americana. Mem. Inst. Oswaldo Crux, 73: 247-253, 1982.

29. VILLALOBOS, G.J.; CAMINOS, N.V.; DURÁN, T.F. \& ANCIANI, I.D. Leishmaniasis tegumentaria americana: aspectos clínicos, parasitológicos e imunoalérgicos em Zipayare, Estado Zulia. Venezuela. Kasmera, 15: 147-166, 1987

30. YAMAMOTO, Y.I.; CENEVIVA, A.C. \& MOURA, R.A.A. - Leishmaniose cutâneo-mucosa: reaçāo de hemaglutinação passiva e de contraimunoeletroforese com antígeno de Leishmania braziliensis. Rev. Farm. Bioquim. Univ. S. Paulo, 17: 123-132, 1981.

31. WALTON, B.C.; BROOKS, W.H. \& ARJONA, I. Serodiagnosis of American leishmaniasis by indiret antibody test. Amer. J. trop. Med. Hyg., 21: 296-299, 1972.

Recebido para publicação em 28/6/1991 Aceito para publicação em 13/3/1992 